\title{
Bosonization and Fermionization of the Superstring Oscillators
}

\author{
Davoud Kamani \\ Faculty of Physics, Amir Kabir University of Technology \\ P.O.Box: 15875-4413, Tehran, Iran \\ e-mail:kamani@aut.ac.ir
}

\begin{abstract}
In this manuscript we consider the transformations of the oscillators of the bosonic fields of the superstring in terms of the fermions oscillators and vice versa. We demand the exchange of the commutation and anti-commutation relations of the oscillators. Therefore, we obtain some conditions on the Grassmannian matrices that appear in these transformations. We observe that there are several methods to obtain these conditions. In addition, adjoints of the matrix elements and $T$-duality of these matrices will be obtained. The effects of this bosonization and fermionization on the mass operators and on some massless states will be studied. The covariant formalism will be used and hence we consider both the matter parts and the ghost parts of the superstring theory.
\end{abstract}

PACS: $11.25 .-\mathrm{w}$

Keywords: Bosonization; Fermionization; Superstring. 


\section{Introduction}

There have been some efforts to understand bosonization and fermionization of various models. In the method of bosonization, the fermions of a theory are expressed in terms of bosons. This provides a powerful non-perturbative tool for investigations in quantum field theory [1]. The extension of this idea can be seen in the Refs. $[2,3,4,5]$. Also see [6] and references therein. The bosonization formalism includes the topics like smooth bosonization [3], connection between dual transformations and bosonization [4], bosonization in higher dimensions [5], functional integral method in bosonization and so on. In the method of fermionization, one can eliminate the bosons in favor of fermions. For example, this method has applications in the heterotic string [7]. In fact, many phenomena that are difficult to understand in the usual language take simple forms in the transformed language.

We consider the superstring theory. The transformations of the worldsheet bosonic fields in terms of the fermionic fields of the worldsheet (and vice versa) are not consistent. These inconsistencies appear in the level of the superstring oscillators. In other words, in this level the transformation matrices should depend on the mode numbers. Therefore, we concentrate on the oscillators of the worldsheet fields. Since calculations and results for the closed and open superstrings are similar, we only consider the closed superstring theory.

We transform the oscillators of the bosonic fields to the fermions oscillators and vice versa. This bosonization and fermionization will be done simultaneously. These exchanges will be done for both the matter parts and the ghost parts of the R-R and NS-NS sectors of the superstring theory. Demanding the exchange of the commutation and anti-commutation relations of the oscillators, we obtain some conditions on the transformation matrices. Therefore, successive transformations (i.e., rebosonization and refermionization) lead to the trivial or orthogonal rotations of the oscillators. The elements of the matrices are Grassmannian variables and carry the mode numbers. The $T$-duality of these matrices and the adjoints of their elements will be obtained. The effects of this bosonization-fermionization on the mass operators and on some massless states also will be studied.

The exchange of the bosons and fermions oscillators can be applied on any quantity which is made from the superstring oscillators. For example, one can apply them on the Virasoro operators, the BRST charge, boundary state of a D p-brane, string field theory and so on.

This paper is organized as follows. In section 2, we shall study the effects of our bosonization-fermionization in the matter part of the R-R sector. In section 3, we shall do the same for the matter part of the NS-NS sector. In section 4, the T-duality of the transformation matrices will be obtained. In section 5 , the bosonization-fermionization in 
the ghost parts of the superstring theory will be discussed. In section 6 , the adjoints of the matrix elements of the transformation matrices will be obtained.

\section{Exchange of oscillators in the matter part of the R-R sector}

\subsection{Conditions on the transformation matrices}

The quantization of the superstring worldsheet fields leads to the following equations

$$
\begin{aligned}
& {\left[\alpha_{m}^{\mu}, \alpha_{n}^{\nu}\right]=\left[\tilde{\alpha}_{m}^{\mu}, \tilde{\alpha}_{n}^{\nu}\right]=m \eta^{\mu \nu} \delta_{m+n, 0}} \\
& {\left[\alpha_{m}^{\mu}, \tilde{\alpha}_{n}^{\nu}\right]=0} \\
& {\left[x^{\mu}, p^{\nu}\right]=i \eta^{\mu \nu}}
\end{aligned}
$$

for the bosonic part, and

$$
\begin{aligned}
& \left\{d_{m}^{\mu}, d_{n}^{\nu}\right\}=\left\{\tilde{d}_{m}^{\mu}, \tilde{d}_{n}^{\nu}\right\}=\eta^{\mu \nu} \delta_{m+n, 0} \\
& \left\{d_{m}^{\mu}, \tilde{d}_{n}^{\nu}\right\}=0
\end{aligned}
$$

for the fermionic part.

Now we transform the bosons oscillators as in the following

$$
\begin{aligned}
& \alpha_{n}^{\mu} \longrightarrow a_{n}^{\mu}=\left(\lambda_{n}\right)^{\mu}{ }_{\nu} d_{n}^{\nu}, \\
& \tilde{\alpha}_{n}^{\mu} \longrightarrow \tilde{a}_{n}^{\mu}=\left(\tilde{\lambda}_{n}\right)^{\mu}{ }_{\nu} \tilde{d}_{n}^{\nu},
\end{aligned}
$$

where $n$ is a nonzero integer. For the zero modes we introduce the transformations

$$
\begin{aligned}
& l p^{\mu} \longrightarrow \lambda^{\mu}{ }_{\nu} d_{0}^{\nu}+\tilde{\lambda}^{\mu}{ }_{\nu} \tilde{d}_{0}^{\nu}, \\
& x^{\mu} \longrightarrow \chi^{\mu}{ }_{\nu} d_{0}^{\nu}+\tilde{\chi}^{\mu}{ }_{\nu} \tilde{d}_{0}^{\nu}
\end{aligned}
$$

where $l=\sqrt{2 \alpha^{\prime}}$. Assume that the spacetime is non-compact therefore, $\alpha_{0}^{\mu}=\tilde{\alpha}_{0}^{\mu}=\frac{1}{2} l p^{\mu}$. The elements of the matrices $\lambda_{n}, \tilde{\lambda}_{n}, \lambda, \tilde{\lambda}, \chi$ and $\tilde{\chi}$ are Grassmannian variables.

Applying the transformations (3) and (4) in the equations (1) and using the equations (2), give the following conditions on the transformation matrices

$$
\lambda_{n} \lambda_{-n}^{T}=\tilde{\lambda}_{n} \tilde{\lambda}_{-n}^{T}=-n \mathbf{1}
$$


for $n \neq 0$. For the zero-mode matrices there are

$$
\begin{aligned}
& \chi \lambda^{T}+\tilde{\chi} \tilde{\lambda}^{T}=-i l \mathbf{1} \\
& \lambda \lambda^{T}+\tilde{\lambda} \tilde{\lambda}^{T}=0 \\
& \chi \chi^{T}+\tilde{\chi} \tilde{\chi}^{T}=0
\end{aligned}
$$

The first condition comes from the noncommutativity of $x^{\mu}$ and $p^{\nu}$, while the second and the third are results of $\left[p^{\mu}, p^{\nu}\right]=0$ and $\left[x^{\mu}, x^{\nu}\right]=0$, respectively.

Now consider the following mappings for the fermions oscillators in terms of the bosons oscillators

$$
\begin{aligned}
d_{n}^{\mu} \longrightarrow D_{n}^{\prime \mu} & =-\frac{1}{n}\left(\lambda_{-n}^{T}\right)^{\mu}{ }_{\nu} \alpha_{n}^{\nu}, \\
\tilde{d}_{n}^{\mu} \longrightarrow \tilde{D}_{n}^{\prime \mu} & =-\frac{1}{n}\left(\tilde{\lambda}_{-n}^{T}\right)^{\mu}{ }_{\nu} \tilde{\alpha}_{n}^{\nu}
\end{aligned}
$$

for $n \neq 0$. The zero modes have the mappings

$$
\begin{aligned}
& d_{0}^{\mu} \longrightarrow \frac{i}{l}\left(-l\left(\chi^{T}\right)^{\mu}{ }_{\nu} p^{\nu}+\left(\lambda^{T}\right)^{\mu}{ }_{\nu} x^{\nu}\right), \\
& \tilde{d}_{0}^{\mu} \longrightarrow \frac{i}{l}\left(-l\left(\tilde{\chi}^{T}\right)^{\mu}{ }_{\nu} p^{\nu}+\left(\tilde{\lambda}^{T}\right)^{\mu}{ }_{\nu} x^{\nu}\right) .
\end{aligned}
$$

Since two successive transformations should be trivial, we require these forms of transformations for the fermions oscillators. See the equations (20) and (21). Introducing the bosonizations (7) and (8) in the equations (2) and using the equations (1) lead to the conditions

$$
\lambda_{n}^{T} \lambda_{-n}=\tilde{\lambda}_{n}^{T} \tilde{\lambda}_{-n}=n \mathbf{1}
$$

for the nonzero-mode matrices, and

$$
\begin{aligned}
& \chi^{T} \lambda-\lambda^{T} \chi=i l \mathbf{1}, \\
& \chi^{T} \tilde{\lambda}-\lambda^{T} \tilde{\chi}=0, \\
& \tilde{\chi}^{T} \tilde{\lambda}-\tilde{\lambda}^{T} \tilde{\chi}=i l \mathbf{1},
\end{aligned}
$$

for the zero-mode matrices. In fact, the conditions (9) and (10) are not independent of (5) and (6). The commutators $\left[\alpha_{0}^{\mu}, \alpha_{n}^{\nu}\right]$ and $\left[\alpha_{0}^{\mu}, \tilde{\alpha}_{n}^{\nu}\right]$ and the anti-commutators $\left\{d_{0}^{\mu}, d_{n}^{\nu}\right\}$ and $\left\{d_{0}^{\mu}, \tilde{d}_{n}^{\nu}\right\}$, for $n \neq 0$, are zero. They do not put new restrictions on the transformation matrices. We shall see that the conditions (5), (6), (9) and (10) also can be obtained by various methods. 
It is possible to apply only the bosonization or the fermionization. In this case the transformed theory has one kind of the commuting or anti-commuting oscillators. However, we consider both of them. Therefore, on any expression of the superstring oscillators, the mappings of bosonization and fermionization will apply simultaneously.

\subsection{Successive transformations}

We replaced the oscillators $\alpha_{n}^{\mu}, \tilde{\alpha}_{n}^{\mu}, d_{n}^{\mu}$ and $\tilde{d}_{n}^{\mu}$ with $a_{n}^{\mu}, \tilde{a}_{n}^{\mu}, D_{n}^{\prime \mu}$ and $\tilde{D}_{n}^{\prime \mu}$, respectively. The conditions (5) and (9) imply that the oscillators $\left\{a_{n}^{\mu}, \tilde{a}_{n}^{\mu}, D_{n}^{\prime \mu}, \tilde{D}_{n}^{\prime \mu}\right\}$ also obey the equations (1) and (2). Now perform transformations on the new oscillators

$$
\begin{gathered}
a_{n}^{\mu} \longrightarrow A_{n}^{\mu}=\left(\bar{\lambda}_{n}\right)^{\mu}{ }_{\nu} D_{n}^{\prime \nu}, \\
\tilde{a}_{n}^{\mu} \longrightarrow \tilde{A}_{n}^{\mu}=\left(\tilde{\bar{\lambda}}_{n}\right)^{\mu}{ }_{\nu} \tilde{D}_{n}^{\prime \nu}, \\
D_{n}^{\prime \mu} \longrightarrow D_{n}^{\mu}=-\frac{1}{n}\left(\bar{\lambda}_{-n}^{T}\right)^{\mu}{ }_{\nu} a_{n}^{\nu}, \\
\tilde{D}_{n}^{\prime \mu} \longrightarrow \tilde{D}_{n}^{\mu}=-\frac{1}{n}\left(\tilde{\bar{\lambda}}_{-n}^{T}\right)_{\nu}^{\mu} \tilde{a}_{n}^{\nu} .
\end{gathered}
$$

The exchange of the commutation and anti-commutation relations of the transformed oscillators $\left\{a_{n}^{\mu}, \tilde{a}_{n}^{\mu}, D_{n}^{\prime \mu}, \tilde{D}_{n}^{\prime \mu}\right\}$ under the above transformations leads to the following conditions for the matrices $\bar{\lambda}_{n}$ and $\tilde{\bar{\lambda}}_{n}$,

$$
\begin{aligned}
& \bar{\lambda}_{n} \bar{\lambda}_{-n}^{T}=\tilde{\bar{\lambda}}_{n} \tilde{\bar{\lambda}}_{-n}^{T}=-n \mathbf{1} \\
& \bar{\lambda}_{n}^{T} \bar{\lambda}_{-n}=\tilde{\bar{\lambda}}_{n}^{T} \tilde{\bar{\lambda}}_{-n}=n \mathbf{1} .
\end{aligned}
$$

These conditions are analog of the equations (5) and (9).

Combination of the transformations (3) and (11) gives

$$
\begin{aligned}
\alpha_{n}^{\mu} \longrightarrow A_{n}^{\mu} & =-\frac{1}{n}\left(\bar{\lambda}_{n} \lambda_{-n}^{T}\right)^{\mu}{ }_{\nu} \alpha_{n}^{\nu}, \\
\tilde{\alpha}_{n}^{\mu} \longrightarrow \tilde{A}_{n}^{\mu} & =-\frac{1}{n}\left(\tilde{\bar{\lambda}}_{n} \tilde{\lambda}_{-n}^{T}\right)^{\mu}{ }_{\nu} \tilde{\alpha}_{n}^{\nu},
\end{aligned}
$$

where the definitions of $D_{n}^{\prime \mu}$ and $\tilde{D}_{n}^{\prime \mu}$ from (7) were used. In fact, these are rebosonization forms of the oscillators $\alpha_{n}^{\mu}$ and $\tilde{\alpha}_{n}^{\mu}$. Now define the matrices $\Lambda_{n}$ and $\tilde{\Lambda}_{n}$ as

$$
\begin{aligned}
& \Lambda_{n}=-\frac{1}{n}\left(\bar{\lambda}_{n} \lambda_{-n}^{T}\right), \\
& \tilde{\Lambda}_{n}=-\frac{1}{n}\left(\tilde{\bar{\lambda}}_{n} \tilde{\lambda}_{-n}^{T}\right) .
\end{aligned}
$$


These matrices satisfy the identities

$$
\Lambda_{n}^{T} \Lambda_{-n}=\Lambda_{-n} \Lambda_{n}^{T}=\tilde{\Lambda}_{n}^{T} \tilde{\Lambda}_{-n}=\tilde{\Lambda}_{-n} \tilde{\Lambda}_{n}^{T}=\mathbf{1}
$$

which can be interpreted as orthogonality-like equations.

Using the definitions of $a_{n}^{\mu}$ and $\tilde{a}_{n}^{\mu}$ from (3), the equations (7) and (12) lead to the transformations

$$
\begin{aligned}
d_{n}^{\mu} \longrightarrow D_{n}^{\mu} & =-\frac{1}{n}\left(\bar{\lambda}_{-n}^{T} \lambda_{n}\right)^{\mu}{ }_{\nu} d_{n}^{\nu} \\
\tilde{d}_{n}^{\mu} \longrightarrow \tilde{D}_{n}^{\mu} & =-\frac{1}{n}\left(\tilde{\bar{\lambda}}_{-n}^{T} \tilde{\lambda}_{n}\right)^{\mu}{ }_{\nu} \tilde{d}_{n}^{\nu}
\end{aligned}
$$

These are refermionizations of $d_{n}^{\mu}$ and $\tilde{d}_{n}^{\mu}$. The matrices

$$
\begin{aligned}
& \Gamma_{n}=-\frac{1}{n}\left(\bar{\lambda}_{-n}^{T} \lambda_{n}\right), \\
& \tilde{\Gamma}_{n}=-\frac{1}{n}\left(\tilde{\bar{\lambda}}_{-n}^{T} \tilde{\lambda}_{n}\right),
\end{aligned}
$$

which appeared in the rotations (17), also satisfy the orthogonality-like equations

$$
\Gamma_{n}^{T} \Gamma_{-n}=\Gamma_{-n} \Gamma_{n}^{T}=\tilde{\Gamma}_{n}^{T} \tilde{\Gamma}_{-n}=\tilde{\Gamma}_{-n} \tilde{\Gamma}_{n}^{T}=\mathbf{1}
$$

Similar discussions also hold for the zero modes.

\subsection{Alternatives for the conditions on the matrices}

In the transformations (3) apply the transformations (7). Thus, we obtain (14) with $\bar{\lambda}_{n}=\lambda_{n}$ and $\tilde{\bar{\lambda}}_{n}=\tilde{\lambda}_{n}$,

$$
\begin{gathered}
\alpha_{n}^{\mu} \longrightarrow-\frac{1}{n}\left(\lambda_{n} \lambda_{-n}^{T}\right)^{\mu}{ }_{\nu} \alpha_{n}^{\nu}, \\
\tilde{\alpha}_{n}^{\mu} \longrightarrow-\frac{1}{n}\left(\tilde{\lambda}_{n} \tilde{\lambda}_{-n}^{T}\right)_{\nu}^{\mu}{ }_{\nu} \tilde{\alpha}_{n}^{\nu} .
\end{gathered}
$$

These rebosonizations should be trivial. This triviality imposes the conditions (5) on the matrices $\lambda_{n}$ and $\tilde{\lambda}_{n}$. Similarly, in the transformations (7) introduce the transformations (3). These lead to the special form of the transformations (17),

$$
\begin{aligned}
& d_{n}^{\mu} \longrightarrow-\frac{1}{n}\left(\lambda_{-n}^{T} \lambda_{n}\right)^{\mu}{ }_{\nu} d_{n}^{\nu}, \\
& \tilde{d}_{n}^{\mu} \longrightarrow-\frac{1}{n}\left(\tilde{\lambda}_{-n}^{T} \tilde{\lambda}_{n}\right)^{\mu}{ }_{\nu} \tilde{d}_{n}^{\nu} .
\end{aligned}
$$

Triviality of these refermionizations gives the conditions (9). 
The same is true for the zero modes. That is, application of (8) in (4) leads to the conditions (6). Also application of (4) in (8) imposes the conditions (10).

The mass operator

In the R-R sector, the matter part of the mass operator is

$$
\alpha^{\prime} M_{R}^{2}=\sum_{n=1}^{\infty}\left(\alpha_{-n} \cdot \alpha_{n}+\tilde{\alpha}_{-n} \cdot \tilde{\alpha}_{n}+n\left(d_{-n} \cdot d_{n}+\tilde{d}_{-n} \cdot \tilde{d}_{n}\right)\right)+a_{R} .
$$

Under the mappings (3) and (7) this operator is invariant. In other words, as another alternative, this invariance leads to the conditions (5) and (9) for the matrices $\lambda_{n}$ and $\tilde{\lambda}_{n}$.

The equations (16) guarantee the invariance of the bosonic part of this mass operator under the rotations (14). Similarly, the invariance of the fermionic part, under the rotations (17), can be seen by the equations (19).

\section{Exchange of oscillators in the matter part of the NS- NS sector}

\subsection{Conditions on the transformation matrices}

The quantization of the fermionic fields of the worldsheet gives the following anti-commutation relations

$$
\begin{aligned}
& \left\{b_{r}^{\mu}, b_{s}^{\nu}\right\}=\left\{\tilde{b}_{r}^{\mu}, \tilde{b}_{s}^{\nu}\right\}=\eta^{\mu \nu} \delta_{r+s, 0}, \\
& \left\{b_{r}^{\mu}, \tilde{b}_{s}^{\nu}\right\}=0 .
\end{aligned}
$$

For this sector we consider the transformations

$$
\begin{aligned}
& \alpha_{n}^{\mu} \longrightarrow\left(\theta_{n}\right)^{\mu}{ }_{\nu} b_{r_{n}}^{\nu}, \\
& \tilde{\alpha}_{n}^{\mu} \longrightarrow\left(\tilde{\theta}_{n}\right)^{\mu}{ }_{\nu} \tilde{b}_{r_{n}}^{\nu},
\end{aligned}
$$

where the elements of the matrices $\theta_{n}$ and $\tilde{\theta}_{n}$ are Grassmannian variables and the half-integer $r_{n}$ is defined by

$$
r_{n}=n-\frac{1}{2} \operatorname{sgn}(n), \quad n \neq 0
$$

The function $\operatorname{sgn}(n)$ denotes the $\operatorname{sign}$ of $n$. This implies $r_{n}+r_{-n}=0$. Substitute the transformations (24) in the equations (1) and also use (23), we obtain the conditions

$$
\theta_{n} \theta_{-n}^{T}=\tilde{\theta}_{n} \tilde{\theta}_{-n}^{T}=-n \mathbf{1}
$$


The transformations of the oscillators of the fermions have the forms

$$
\begin{aligned}
& b_{r_{n}}^{\mu} \longrightarrow-\frac{1}{n}\left(\theta_{-n}^{T}\right)^{\mu}{ }_{\nu} \alpha_{n}^{\nu}, \\
& \tilde{b}_{r_{n}}^{\mu} \longrightarrow-\frac{1}{n}\left(\tilde{\theta}_{-n}^{T}\right)^{\mu}{ }_{\nu} \tilde{\alpha}_{n}^{\nu} .
\end{aligned}
$$

Under these mappings the equations (1) and (23) put the following restrictions on the matrices $\theta_{n}$ and $\tilde{\theta}_{n}$,

$$
\theta_{n}^{T} \theta_{-n}=\tilde{\theta}_{n}^{T} \tilde{\theta}_{-n}=n \mathbf{1}
$$

These are not independent of (26). Combination of the transformations (24) and (27) also gives the conditions (26) and (28). The result is analog of the rotations (20) and (21).

According to the equations (1) and (23) the bosonization-fermionization in this sector require the equality $\delta_{r_{m}+r_{n}, 0}=\delta_{m+n, 0}$. Since $m$ and $n$ are nonzero integers, for the various cases, i.e., $m, n \geq 1, m, n \leq-1$ and $m \geq 1, n \leq-1$ ( or $m \leq-1, n \geq 1$ ) this equality holds.

The zero modes $x^{\mu}$ and $p^{\mu}$ can be fermionized in terms of the oscillators $\left\{b_{1 / 2}^{\mu}, b_{-1 / 2}^{\mu}\right\}$ or $\left\{\tilde{b}_{1 / 2}^{\mu}, \tilde{b}_{-1 / 2}^{\mu}\right\}$. For example, for the first set we can write

$$
\left(\begin{array}{c}
x \\
l p
\end{array}\right) \longrightarrow\left(\begin{array}{cc}
\theta_{+} & \theta_{-} \\
\lambda_{+} & \lambda_{-}
\end{array}\right)\left(\begin{array}{c}
b_{1 / 2} \\
b_{-1 / 2}
\end{array}\right),
$$

where $\theta_{+}, \theta_{-}, \lambda_{+}$and $\lambda_{-}$are $10 \times 10$ matrices with the anti-commuting elements. The commutation relations of $x^{\mu}$ and $p^{\mu}$ put the following conditions on the matrices

$$
\begin{aligned}
& \theta_{+} \lambda_{-}^{T}+\theta_{-} \lambda_{+}^{T}=-i l \mathbf{1}, \\
& \theta_{+} \theta_{-}^{T}+\theta_{-} \theta_{+}^{T}=0, \\
& \lambda_{+} \lambda_{-}^{T}+\lambda_{-} \lambda_{+}^{T}=0 .
\end{aligned}
$$

These equations correspond to the relations $\left[x^{\mu}, p^{\nu}\right]=i \eta^{\mu \nu},\left[x^{\mu}, x^{\nu}\right]=0$ and $\left[p^{\mu}, p^{\nu}\right]=0$, respectively.

The mass operator for this sector is

$$
\alpha^{\prime} M_{N S}^{2}=\sum_{n=1}^{\infty}\left(\alpha_{-n} \cdot \alpha_{n}+\tilde{\alpha}_{-n} \cdot \tilde{\alpha}_{n}+r_{n}\left(b_{-r_{n}} \cdot b_{r_{n}}+\tilde{b}_{-r_{n}} \cdot \tilde{b}_{r_{n}}\right)\right)+a_{N S} .
$$

This operator under the transformations (24) and (27) takes the form

$$
\alpha^{\prime} \bar{M}_{N S}^{2}=\sum_{n=1}^{\infty}\left(\left(1-\frac{1}{2 n}\right)\left(\alpha_{-n} \cdot \alpha_{n}+\tilde{\alpha}_{-n} \cdot \tilde{\alpha}_{n}\right)+n\left(b_{-r_{n}} \cdot b_{r_{n}}+\tilde{b}_{-r_{n}} \cdot \tilde{b}_{r_{n}}\right)\right)+a_{N S} .
$$


The mass operator (32) belongs to the transformed theory. Thus, it acts on the transformed states.

Note that the transformation of the superstring theory gives a new theory for the superstring. Under this transformation the algebras of the oscillators remain invariant. This is similar to the superstring theory and its $T$-dual theory that the mass operator is common between them.

The successive transformations with the matrices $\theta_{n}, \tilde{\theta}_{n}, \bar{\theta}_{n}$ and $\tilde{\bar{\theta}}_{n}$ produce rotations which are analog of (14) and (17),

$$
\begin{gathered}
\alpha_{n}^{\mu} \longrightarrow-\frac{1}{n}\left(\bar{\theta}_{n} \theta_{-n}^{T}\right)^{\mu}{ }_{\nu} \alpha_{n}^{\nu}, \\
\tilde{\alpha}_{n}^{\mu} \longrightarrow-\frac{1}{n}\left(\tilde{\bar{\theta}}_{n} \tilde{\theta}_{-n}^{T}\right)^{\mu}{ }_{\nu} \tilde{\alpha}_{n}^{\nu}, \\
b_{r_{n}}^{\mu} \longrightarrow-\frac{1}{n}\left(\bar{\theta}_{-n}^{T} \theta_{n}\right)^{\mu}{ }_{\nu} b_{r_{n}}^{\nu}, \\
\tilde{b}_{r_{n}}^{\mu} \longrightarrow-\frac{1}{n}\left(\tilde{\bar{\theta}}_{-n}^{T} \tilde{\theta}_{n}\right)^{\mu}{ }_{\nu} \tilde{b}_{r_{n}}^{\nu} .
\end{gathered}
$$

The matrices in these rotations are orthogonal-like. Therefore, the bosonic and the fermionic parts of the mass operator (31), under these rotations, are invariant.

\subsection{Examples}

\subsubsection{A massless state}

Consider the following massless state with the momentum $k^{\mu}$,

$$
\left|\phi^{\mu}\right\rangle=b_{-1 / 2}^{\mu}|k ; Z\rangle
$$

If the state $|Z\rangle$ is the NS vacuum, the above state belongs to the open superstring spectrum. Therefore, it describes a gauge field. If $|Z\rangle$ is the Ramond vacuum, the state (33) is a massless state of closed superstring.

Under the bosonization, it changes as

$$
\left|\phi^{\mu}\right\rangle \longrightarrow\left|\bar{\phi}^{\mu}\right\rangle \equiv\left(\theta_{1}^{T}\right)^{\mu}{ }_{\nu} \alpha_{-1}^{\nu}|k ; Z\rangle
$$

The state $\alpha_{-1}^{\mu}|k ; Z\rangle$ is a massive vector field in the bosonic part of the superstring theory. Let $\varepsilon_{\mu}$ be polarization vector of the state (35). Thus, the vector state $\alpha_{-1}^{\mu}|k ; Z\rangle$ has the polarization

$$
\epsilon_{\nu}=\varepsilon_{\mu}\left(\theta_{1}^{T}\right)_{\nu}^{\mu}
$$


Since the components of $\varepsilon_{\mu}$ are commuting numbers, the components of $\epsilon_{\mu}$ are anti-commuting numbers. The mass of the state $\left|\phi^{\mu}\right\rangle$ should be calculated by the operator (31), while the operator (32) determines the mass of $\left|\bar{\phi}^{\mu}\right\rangle$,

$$
\begin{aligned}
\alpha^{\prime} M_{N S}^{2}\left|\phi^{\mu}\right\rangle & =\left(\frac{1}{2}+a_{N S}\right)\left|\phi^{\mu}\right\rangle, \\
\alpha^{\prime} \bar{M}_{N S}^{2}\left|\bar{\phi}^{\mu}\right\rangle & =\left(\frac{1}{2}+a_{N S}\right)\left|\bar{\phi}^{\mu}\right\rangle .
\end{aligned}
$$

Under the bosonization-fermionization we have the exchange $\left|\phi^{\mu}\right\rangle \longleftrightarrow\left|\bar{\phi}^{\mu}\right\rangle$. The exchange also occurs in the mass operators $M_{N S}^{2} \longleftrightarrow \bar{M}_{N S}^{2}$. Including the ghost parts, the normal ordering constant is $a_{N S}=-\frac{1}{2}$.

\subsubsection{Presence of a $\mathrm{D} p$-brane}

Consider a D $p$-brane that its worldvolume is along the directions $\left\{X^{\alpha}\right\}$. Let the set $\left\{X^{i}\right\}$ denote the coordinates which are perpendicular to the worldvolume of the brane. The massless states

$$
b_{-1 / 2}^{\alpha}|k\rangle, \quad b_{-1 / 2}^{i}|k\rangle
$$

are gauge field and collective coordinates of this D $p$-brane. Under the transformations (27) these states transform as in the following

$$
\begin{aligned}
& b_{-1 / 2}^{\alpha}|k\rangle \longrightarrow\left(\theta_{1}^{T}\right)^{\alpha}{ }_{\beta} \alpha_{-1}^{\beta}|k\rangle+\left(\theta_{1}^{T}\right)^{\alpha}{ }_{j} \alpha_{-1}^{j}|k\rangle, \\
& b_{-1 / 2}^{i}|k\rangle \longrightarrow\left(\theta_{1}^{T}\right)^{i}{ }_{\beta} \alpha_{-1}^{\beta}|k\rangle+\left(\theta_{1}^{T}\right)^{i}{ }_{j} \alpha_{-1}^{j}|k\rangle .
\end{aligned}
$$

That is, the transformed versions of the gauge field and the collective coordinates of the brane have expressions in terms of the massive vector field and collective coordinates of the bosonic part of the theory. Note that by the operator (32), the right-hand-sides of (40) also are massless, as expected.

\subsubsection{The metric and antisymmetric tensor}

The massless state of the NS-NS sector

$$
\left|\phi^{\mu \nu}, \eta\right\rangle=\left(b_{-1 / 2}^{\mu} \tilde{b}_{-1 / 2}^{\nu}+\eta b_{-1 / 2}^{\nu} \tilde{b}_{-1 / 2}^{\mu}\right)|k\rangle
$$

describes the metric $G_{\mu \nu}($ for $\eta=1)$ and the antisymmetric tensor $B_{\mu \nu}$ (for $\left.\eta=-1\right)$. After bosonization, it transforms to the state

$$
\left|\bar{\phi}^{\mu \nu}, \eta\right\rangle=\left(\left(\theta_{1}^{T} W \tilde{\theta}_{1}\right)^{\mu \nu}+\eta\left(\theta_{1}^{T} W \tilde{\theta}_{1}\right)^{\nu \mu}\right)|k\rangle
$$


where the matrix $W^{\mu \nu}$ has the definition

$$
W^{\mu \nu}=\alpha_{-1}^{\mu} \tilde{\alpha}_{-1}^{\nu}
$$

Using the mass operator (32), we observe that the state (42) is massless.

\section{$4 \quad T$-duality of the transformation matrices}

Let the direction $X^{\mu_{0}}$ be compact. Therefore, the oscillators $\left\{\alpha_{n}^{\mu^{\prime}}, d_{n}^{\mu^{\prime}}, b_{r}^{\mu^{\prime}}, \tilde{\alpha}_{n}^{\mu}, \tilde{d}_{n}^{\mu}, \tilde{b}_{r}^{\mu}\right\}$ with $\mu^{\prime} \neq \mu_{0}$, under the $T$-duality do not change, while the oscillators $\left\{\alpha_{n}^{\mu_{0}}, d_{n}^{\mu_{0}}, b_{r}^{\mu_{0}}\right\}$ transform as

$$
\begin{aligned}
& \alpha_{n}^{\mu_{0}} \longrightarrow-\alpha_{n}^{\mu_{0}}, \\
& d_{n}^{\mu_{0}} \longrightarrow-d_{n}^{\mu_{0}}, \\
& b_{r}^{\mu_{0}} \longrightarrow-b_{r}^{\mu_{0}} .
\end{aligned}
$$

On all oscillators of the superstring, exchanges of the oscillators accompanied by the $T$ duality transformations can be applied successively. For example, let study the R-R sector in detail.

On the oscillator $\alpha_{n}^{\mu_{0}}$ apply $T$-duality and then fermionization. These give

$$
\alpha_{n}^{\mu_{0}} \longrightarrow-\alpha_{n}^{\mu_{0}} \longrightarrow-\left(\lambda_{n}\right)_{\mu_{0}}^{\mu_{0}} d_{n}^{\mu_{0}}-\left(\lambda_{n}\right)_{\mu^{\prime}}^{\mu_{0}} d_{n}^{\mu^{\prime}}
$$

Now perform fermionization and then T-duality

$$
\alpha_{n}^{\mu_{0}} \longrightarrow\left(\lambda_{n}\right)_{\mu_{0}}^{\mu_{0}} d_{n}^{\mu_{0}}+\left(\lambda_{n}\right)_{\mu^{\prime}}^{\mu_{0}} d_{n}^{\mu^{\prime}} \longrightarrow-\left(\lambda_{n}^{\prime}\right)_{\mu_{0}}^{\mu_{0}} d_{n}^{\mu_{0}}+\left(\lambda_{n}^{\prime}\right)_{\mu^{\prime}}^{\mu_{0}} d_{n}^{\mu^{\prime}}
$$

where the matrix $\lambda_{n}^{\prime}$ is the $T$-dual of $\lambda_{n}$. We demand that the final results to be equal. Thus, we obtain

$$
\begin{aligned}
& \left(\lambda_{n}^{\prime}\right)_{\mu_{0}}^{\mu_{0}}=\left(\lambda_{n}\right)_{\mu_{0}}^{\mu_{0}}, \\
& \left(\lambda_{n}^{\prime}\right)_{\mu^{\prime}}^{\mu_{0}}=-\left(\lambda_{n}\right)_{\mu^{\prime}}^{\mu_{0}} .
\end{aligned}
$$

Now apply the processes $T$-duality-fermionization on the oscillator $\alpha_{n}^{\mu^{\prime}}$. These give the other elements of the matrix $\lambda_{n}^{\prime}$,

$$
\begin{aligned}
& \left(\lambda_{n}^{\prime}\right)_{\mu_{0}}^{\mu^{\prime}}=-\left(\lambda_{n}\right)_{\mu_{0}}^{\mu^{\prime}}, \\
& \left(\lambda_{n}^{\prime}\right)_{\nu^{\prime}}^{\mu^{\prime}}=\left(\lambda_{n}\right)_{\nu^{\prime}}^{\mu^{\prime}} .
\end{aligned}
$$

The equations (47) and (48) completely determine all elements of the $T$-dual matrix $\lambda_{n}^{\prime}$. Note that if we apply the $T$-duality-bosonization processes on the fermions oscillators $d_{n}^{\mu_{0}}$ 
and $d_{n}^{\mu^{\prime}}$, we obtain the same result for the matrix $\lambda_{n}^{\prime}$. For the oscillators of the left-moving fields the corresponding matrices under the $T$-duality do not change, i.e.,

$$
\left(\tilde{\lambda}_{n}^{\prime}\right)_{\nu}^{\mu}=\left(\tilde{\lambda}_{n}\right)_{\nu}^{\mu}
$$

The $T$-dualities of the zero modes $p^{\mu_{0}}=p_{L}^{\mu_{0}}+p_{R}^{\mu_{0}}$ and $x^{\mu_{0}}=x_{L}^{\mu_{0}}+x_{R}^{\mu_{0}}$ are $\frac{1}{\alpha^{\prime}} L^{\mu_{0}}=p_{L}^{\mu_{0}}-p_{R}^{\mu_{0}}$ and $x^{\prime \mu_{0}}=x_{L}^{\mu_{0}}-x_{R}^{\mu_{0}}$, respectively. Under the fermionization we have

$$
\begin{aligned}
& \frac{l}{\alpha^{\prime}} L^{\mu_{0}} \longrightarrow-\lambda_{\nu}^{\mu_{0}} d_{0}^{\nu}+\tilde{\lambda}_{\nu}^{\mu_{0}} \tilde{d}_{0}^{\nu}, \\
& x^{\mu_{0}} \longrightarrow-\chi_{\nu}^{\mu_{0}} d_{0}^{\nu}+\tilde{\chi}_{\nu}^{\mu_{0}} \tilde{d}_{0}^{\nu} .
\end{aligned}
$$

Note that $p^{\mu^{\prime}}$ and $x^{\mu^{\prime}}$ under the $T$-duality are invariant. Therefore, introducing the processes $T$-duality-fermionization on $p^{\mu_{0}}, p^{\mu^{\prime}}, x^{\mu_{0}}$ and $x^{\mu^{\prime}}$, we observe that the $T$-dual versions of the matrices $\lambda, \tilde{\lambda}, \chi$ and $\tilde{\chi}$ also obey the equations (47)-(49).

In the same way, for the NS-NS sector the transformation matrices under the $T$-duality have the following elements

$$
\begin{aligned}
& \left(\theta_{n}^{\prime}\right)_{\mu_{0}}^{\mu_{0}}=\left(\theta_{n}\right)_{\mu_{0}}^{\mu_{0},} \\
& \left(\theta_{n}^{\prime}\right)_{\mu^{\prime}}^{\mu_{0}}=-\left(\theta_{n}\right)_{\mu^{\prime}}^{\mu_{0}}, \\
& \left(\theta_{n}^{\prime}\right)^{\mu^{\prime}{ }_{\mu_{0}}}=-\left(\theta_{n}\right)^{\mu^{\prime}}{ }_{\mu_{0}}, \\
& \left(\theta_{n}^{\prime}\right)_{\nu^{\prime}}^{\mu^{\prime}}=\left(\theta_{n}\right)^{\mu^{\prime}}{ }_{\nu^{\prime}}, \\
& \left(\tilde{\theta}_{n}^{\prime}\right)^{\mu}{ }_{\nu}=\left(\tilde{\theta}_{n}\right)^{\mu}{ }_{\nu} .
\end{aligned}
$$

In other words, the T-dualities of the transformation matrices in the R-R and NS-NS sectors obey the same rule.

\section{Exchange of oscillators in the ghost parts}

\subsection{The ghosts of the R-R sector}

The oscillators of the conformal ghosts and super-conformal ghosts are $\left\{b_{n}, c_{n}, \tilde{b}_{n}, \tilde{c}_{n}\right\}$ and $\left\{\beta_{n}, \gamma_{n}, \tilde{\beta}_{n}, \tilde{\gamma}_{n}\right\}$, respectively. Define the two-component vectors $B_{n}^{i}, \tilde{B}_{n}^{i}, \eta_{n}^{i}$ and $\tilde{\eta}_{n}^{i}$ with $i \in\{1,2\}$ as in the following

$$
\begin{aligned}
& B_{n}^{1}=b_{n}, B_{n}^{2}=c_{n} \\
& \tilde{B}_{n}^{1}=\tilde{b}_{n}, \tilde{B}_{n}^{2}=\tilde{c}_{n} \\
& \eta_{n}^{1}=\beta_{n}, \eta_{n}^{2}=\gamma_{n} \\
& \tilde{\eta}_{n}^{1}=\tilde{\beta}_{n}, \tilde{\eta}_{n}^{2}=\tilde{\gamma}_{n} .
\end{aligned}
$$


According to these definitions the commutation and anti-commutation relations of the ghosts oscillators take the forms

$$
\begin{aligned}
{\left[\eta_{m}^{i}, \eta_{n}^{j}\right] } & =\left[\tilde{\eta}_{m}^{i}, \tilde{\eta}_{n}^{j}\right]=\rho^{i j} \delta_{m+n, 0}, \\
{\left[\eta_{m}^{i}, \tilde{\eta}_{n}^{j}\right] } & =0, \\
\left\{B_{m}^{i}, B_{n}^{j}\right\} & =\left\{\tilde{B}_{m}^{i}, \tilde{B}_{n}^{j}\right\}=S^{i j} \delta_{m+n, 0}, \\
\left\{B_{m}^{i}, \tilde{B}_{n}^{j}\right\} & =0,
\end{aligned}
$$

where the symmetric matrix $S$ and the antisymmetric matrix $\rho$ are defined by

$$
S=\left(\begin{array}{ll}
0 & 1 \\
1 & 0
\end{array}\right), \quad \rho=\left(\begin{array}{cc}
0 & -1 \\
1 & 0
\end{array}\right)
$$

We shall use the following properties of these matrices

$$
\rho S+S \rho=0, \quad S^{2}=\mathbf{1}, \quad \rho^{2}=-\mathbf{1} .
$$

For the components of the vectors $\eta_{n}^{i}$ and $\tilde{\eta}_{n}^{i}$ we introduce the following fermionizations

$$
\begin{aligned}
& \eta_{n}^{i} \longrightarrow\left(\Omega_{n}\right)^{i}{ }_{j} B_{n}^{j}, \\
& \tilde{\eta}_{n}^{i} \longrightarrow\left(\tilde{\Omega}_{n}\right)^{i}{ }_{j} \tilde{B}_{n}^{j},
\end{aligned}
$$

where $n$ is any integer mode number, which includes zero. The elements of the $2 \times 2$ matrices $\Omega_{n}$ and $\tilde{\Omega}_{n}$ are Grassmannian quantities. The equations (53) and (54) and the fermionization (57) put the following conditions on the matrices $\Omega_{n}$ and $\tilde{\Omega}_{n}$,

$$
\Omega_{n} S \Omega_{-n}^{T}=\tilde{\Omega}_{n} S \tilde{\Omega}_{-n}^{T}=-\rho
$$

The bosonizations of the components of the vectors $B_{n}^{i}$ and $\tilde{B}_{n}^{i}$ can be considered as

$$
\begin{aligned}
& B_{n}^{i} \longrightarrow\left(S \Omega_{-n}^{T} \rho\right)^{i}{ }_{j} \eta_{n}^{j} \\
& \tilde{B}_{n}^{i} \longrightarrow\left(S \tilde{\Omega}_{-n}^{T} \rho\right)^{i}{ }_{j} \tilde{\eta}_{n}^{j} .
\end{aligned}
$$

Introducing these mappings into (54) and using (53) lead to the conditions

$$
\Omega_{n}^{T} \rho \Omega_{-n}=\tilde{\Omega}_{n}^{T} \rho \tilde{\Omega}_{-n}=S
$$

These conditions are not independent of (58). That is, combinations of the equations (58) and (60) are trivial identities. 
The contributions of the super-conformal ghosts and conformal ghosts to the mass operator are

$$
\begin{aligned}
& \alpha^{\prime} M_{s c g}^{2}=-\sum_{n=1}^{\infty}\left(n\left(\eta_{-n}^{T} \rho \eta_{n}+\tilde{\eta}_{-n}^{T} \rho \tilde{\eta}_{n}\right)\right), \\
& \alpha^{\prime} M_{c g}^{2}=\sum_{n=1}^{\infty}\left(n\left(B_{-n}^{T} S B_{n}+\tilde{B}_{-n}^{T} S \tilde{B}_{n}\right)\right) .
\end{aligned}
$$

Now introduce the transformations (57) and (59) into these operators. According to the conditions (58) and (60) the masses $\alpha^{\prime} M_{c g}^{2}$ and $\alpha^{\prime} M_{s c g}^{2}$ are exchanged. In other words, the quantity $\alpha^{\prime}\left(M_{c g}^{2}+M_{s c g}^{2}\right)$ remains invariant.

\subsection{Successive transformations}

Combine the transformations (57) and (59). We obtain analog of the equations (20) and $(21)$

$$
\begin{aligned}
& \eta_{n}^{i} \longrightarrow\left(\Omega_{n} S \Omega_{-n}^{T} \rho\right)^{i}{ }_{j} \eta_{n}^{j}, \\
& B_{n}^{i} \longrightarrow\left(S \Omega_{-n}^{T} \rho \Omega_{n}\right)^{i}{ }_{j} B_{n}^{j}
\end{aligned}
$$

which are rebosonization and refermionization. Similar transformations also hold for the oscillators of the left-moving fields. Triviality of these transformations leads to the conditions (58) and (60). Inversely, the conditions (58) and (60) imply that the above mappings to be trivial.

Now consider two different successive transformations. The first is distinguished by the

matrices $\Omega_{n}$ and $\tilde{\Omega}_{n}$, and the second by $\bar{\Omega}_{n}$ and $\tilde{\bar{\Omega}}_{n}$. The matrices $\bar{\Omega}_{n}$ and $\tilde{\bar{\Omega}}_{n}$ also satisfy the conditions (58) and (60). Therefore, we have non-trivial rebosonization and refermionization

$$
\begin{aligned}
& \eta_{n}^{i} \longrightarrow\left(\bar{\Omega}_{n} S \Omega_{-n}^{T} \rho\right)^{i}{ }_{j} \eta_{n}^{j} \equiv\left(M_{n}\right)^{i}{ }_{j} \eta_{n}^{j}, \\
& B_{n}^{i} \longrightarrow\left(S \bar{\Omega}_{-n}^{T} \rho \Omega_{n}\right)^{i}{ }_{j} B_{n}^{j} \equiv\left(N_{n}\right)^{i}{ }_{j} B_{n}^{j} .
\end{aligned}
$$

These are analog of the transformations (14), (17), (33) and (34). Similar relations also hold for the left-oscillators. The matrices $M_{n}$ and $N_{n}$ satisfy the identities

$$
\begin{aligned}
& M_{n}^{T} \rho M_{-n}=M_{n} \rho M_{-n}^{T}=\rho, \\
& N_{n}^{T} S N_{-n}=N_{n} S N_{-n}^{T}=S .
\end{aligned}
$$

The meaning of the equations (65) is invariance of the mass (61) under the first transformation of (64). Also the equations (66) imply that the mass (62) under the second transformation of (64) is invariant. 


\subsection{The ghosts of the NS-NS sector}

For the super-conformal ghosts of this sector we have the vectors $\eta_{r}^{i}$ and $\tilde{\eta}_{r}^{i}$, with the components

$$
\begin{array}{ll}
\eta_{r}^{1}=\beta_{r}, & \eta_{r}^{2}=\gamma_{r}, \\
\tilde{\eta}_{r}^{1}=\tilde{\beta}_{r}, & \tilde{\eta}_{r}^{2}=\tilde{\gamma}_{r},
\end{array}
$$

where $r$ is a half-integer number. The commutation relations of these oscillators are

$$
\begin{aligned}
& {\left[\eta_{r}^{i}, \eta_{s}^{j}\right]=\left[\tilde{\eta}_{r}^{i}, \tilde{\eta}_{s}^{j}\right]=\rho^{i j} \delta_{r+s, 0},} \\
& {\left[\eta_{r}^{i}, \tilde{\eta}_{s}^{j}\right]=0 .}
\end{aligned}
$$

Let the transformation matrices for this sector be $\omega_{n}$ and $\tilde{\omega}_{n}$. Therefore, we have the following fermionizations

$$
\begin{aligned}
& \eta_{r_{n}}^{i} \longrightarrow\left(\omega_{n}\right)^{i}{ }_{j} B_{n}^{j}, \\
& \tilde{\eta}_{r_{n}}^{i} \longrightarrow\left(\tilde{\omega}_{n}\right)^{i}{ }_{j} \tilde{B}_{n}^{j},
\end{aligned}
$$

where the half-integer $r_{n}$ has been given by the definition (25). The bosonizations of $B_{n}^{i}$ and $\tilde{B}_{n}^{i}$, for $n \neq 0$, are

$$
\begin{aligned}
& B_{n}^{i} \longrightarrow\left(S \omega_{-n}^{T} \rho\right)^{i}{ }_{j} \eta_{r_{n}}^{j}, \\
& \tilde{B}_{n}^{i} \longrightarrow\left(S \tilde{\omega}_{-n}^{T} \rho\right)^{i}{ }_{j} \tilde{\eta}_{r_{n}}^{j} .
\end{aligned}
$$

According to the mappings (69) and (70), the relations (54) and (68) (or triviality of the successive transformations analog of (63)) give the following conditions on the matrices $\omega_{n}$ and $\tilde{\omega}_{n}$,

$$
\begin{aligned}
& \omega_{n} S \omega_{-n}^{T}=\tilde{\omega}_{n} S \tilde{\omega}_{-n}^{T}=-\rho, \\
& \omega_{n}^{T} \rho \omega_{-n}=\tilde{\omega}_{n}^{T} \rho \tilde{\omega}_{-n}=S .
\end{aligned}
$$

These conditions are not independent of each other. Since the features of the commutation relations (53) and (68) are the same, the matrices $\omega_{n}$ and $\Omega_{n}$ obey the same conditions.

For the zero modes we consider the transformations

$$
\begin{aligned}
& \left(\begin{array}{c}
B_{0}^{1} \\
B_{0}^{2}
\end{array}\right) \longrightarrow S \omega_{0}^{T} \rho\left(\begin{array}{c}
\eta_{1 / 2}^{1} \\
\eta_{-1 / 2}^{2}
\end{array}\right), \\
& \left(\begin{array}{c}
\tilde{B}_{0}^{1} \\
\tilde{B}_{0}^{2}
\end{array}\right) \longrightarrow S \tilde{\omega}_{0}^{T} \rho\left(\begin{array}{c}
\tilde{\eta}_{1 / 2}^{1} \\
\tilde{\eta}_{-1 / 2}^{2}
\end{array}\right) .
\end{aligned}
$$


The commutation relations (54) for $m=n=0$ give the following conditions on the matrices $\omega_{0}$ and $\tilde{\omega}_{0}$,

$$
\omega_{0}^{T} \rho \omega_{0}=\tilde{\omega}_{0}^{T} \rho \tilde{\omega}_{0}=S
$$

The conformal ghost part of the mass operator has been given by (62). The contribution of the super-conformal ghosts is

$$
\alpha^{\prime} M_{s c g}^{2}=-\sum_{n=1}^{\infty}\left(r_{n}\left(\eta_{-r_{n}}^{T} \rho \eta_{r_{n}}+\tilde{\eta}_{-r_{n}}^{T} \rho \tilde{\eta}_{r_{n}}\right)\right) .
$$

The sum of the masses (62) and (74) under the transformations (69) and (70) changes to

$$
\alpha^{\prime}\left(\bar{M}_{c g}^{2}+\bar{M}_{s c g}^{2}\right)=\sum_{n=1}^{\infty}\left(-n\left(\eta_{-r_{n}}^{T} \rho \eta_{r_{n}}+\tilde{\eta}_{-r_{n}}^{T} \rho \tilde{\eta}_{r_{n}}\right)+r_{n}\left(B_{-n}^{T} S B_{n}+\tilde{B}_{-n}^{T} S \tilde{B}_{n}\right)\right)
$$

This operator acts on the transformed states, under (69) and (70).

There are equations analog of (64)-(66) with $\omega_{n}$ and $\bar{\omega}_{n}$ instead of $\Omega_{n}$ and $\bar{\Omega}_{n}$. By these equations, the operators $\alpha^{\prime} M_{c g}^{2}$ and $\alpha^{\prime} M_{s c g}^{2}$ are invariant.

\section{Adjoint of a matrix element}

The adjoint of a superstring oscillator has the property

$$
\left(q_{k}^{\mu}\right)^{\dagger}=q_{-k}^{\mu}
$$

where $q_{k}^{\mu}$ denotes any oscillator of the superstring. For the R-R sector $k$ is integer and for the NS-NS sector it is half-integer. According to this equation, the matrix elements of the transformation matrices have the adjoint as in the following

$$
\begin{aligned}
& {\left[\left(Q_{n}\right)^{\mu}{ }_{\nu}\right]^{\dagger}=\left(Q_{-n}\right)^{\mu}{ }_{\nu},} \\
& Q_{n} \in\left\{\lambda_{n}, \tilde{\lambda}_{n}, \theta_{n}, \tilde{\theta}_{n}, \Omega_{n}, \tilde{\Omega}_{n}, \omega_{n}, \tilde{\omega}_{n}\right\} .
\end{aligned}
$$

This is due to the uniqueness of the bosonization and fermionization of the superstring oscillators. Note that this is adjoint of the matrix elements. It is not the adjoint of the matrices.

\section{Conclusions and summary}

By using the matrices with the Grassmannian elements, we considered transformations of the oscillators of the bosonic fields in terms of the oscillators of the fermionic fields of 
the superstring, and vice versa. The exchange of the commutation and anti-commutation

relations of these oscillators (under the above transformations) puts some conditions on the above matrices. These conditions also can be obtained from the other methods such as triviality of the similar successive transformations.

\section{The matter parts}

In the $\mathrm{R}-\mathrm{R}$ sector the mass operator remains invariant, while in the NS-NS sector it changes. The transformed mass operator acts on the transformed states of the superstring. On the other hand, the transformed theory is a new theory which has common oscillator algebra with the initial theory.

Different successive transformations give (orthogonal) rotations to the oscillators. In other words, these rotations are rebosonization and refermionization. Therefore, under these rotations the bosonic and fermionic parts of the mass operators of the both sectors separately remain invariant.

We obtained the polarization of a massive vector field in terms of the polarization of the superstring gauge field. This vector field comes from the bosonic part of the superstring theory. In addition, transformations of the gauge field and the collective coordinates of a $\mathrm{D} p$-brane were analyzed. The new versions of the spacetime metric and the antisymmetric tensor also were obtained.

The effects of the $T$-duality on the transformation matrices were studied. We observed that in the R-R and NS-NS sectors the T-dualities of the Grassmannian matrices obey the same rule.

\section{The ghost parts}

Since we considered the covariant formulation of the superstring theory, the conformal and the super-conformal ghosts are also analyzed. Therefore, in each sector, by using the corresponding bosonization-fermionization, we obtained the conditions on the transformation matrices and the changes of the mass operators.

We observed that the mass operator in the R-R sector is invariant, while in the NS-NS sector it changes. The matrices of the non-trivial rebosonization and refermionization satisfy some identities. Under these transformations, the mass operators of the conformal ghosts and super-conformal ghosts separately are invariant.

Finally, we saw that the adjoint of a matrix element, similar to the adjoint of an oscillator, changes the sign of the mode number of that matrix element. 


\section{References}

[1] S. Coleman, Phys. Rev. D11(1975)2088; S. Mandelstam, ibid 3026.

[2] E. Witten, Comm. Math. Phys. 92(1984)455; F.A. Schaposnik, Phys. Lett. B356(1995)39.

[3] P.H. Damgaard, H.B. Nielsen and R. Sollacher, Nucl. Phys. B385(1992)227; B414(1994)541; Phys. Lett. B296(1992)132; B322(1994)131; P.H. Damgaard and R. Sollacher, Nucl. Phys. B433(1995)671; N. Banerjee and R. Banerjee Nucl. Phys. B445(1995)516.

[4] C.P. Burgess, C.A. Lutken and F. Quevedo, Phys. Lett. B336(1994)18; C.P. Burgess and F. Quevedo, Nucl. Phys. B421(1994)373; R. Banerjee, Nucl. Phys. B465(1996)157.

[5] E. Fradkin and F.A. Schaposnik, Phys. Lett. B338(1994)253; R. Banerjee, Phys. Lett. B358(1995)297; E.C. Marino, Phys. Lett. B263(1991)63.

[6] A. Dhar, JHEP 0507 (2005) 064, hep-th/0505084.

[7] W. Lerche and A.N. Schellekens, preprint (1987)TH 4925 (CERN); H. Kawai, D.C. Lewellen and S-H Tye, Nucl. Phys. B288(1987)1; I. Antoniadis, C.P. Bachas and C. Kounnas, Nucl. Phys. B289(1987)87. 